\title{
Gustatory event-related potential alterations in olfactory dysfunction patients
}

\author{
Zirong Chen ${ }^{1,2} \cdot$ Chunhua $\mathrm{Hu}^{2} \cdot$ Yaru Zhang $^{3} \cdot \mathrm{Hongbo} \mathrm{Xie}^{1,2} \cdot$ Yongxiang Wei $^{4} \oplus$
}

Received: 27 October 2021 / Accepted: 1 January 2022 / Published online: 2 February 2022

(c) Fondazione Società Italiana di Neurologia 2022

\begin{abstract}
The phenomenon that longstanding impaired olfactory function is associated with the decreased gustatory function was described in present studies, which was seems attributed to mutual chemosensory interactions. And the interaction between olfaction and gustation still needs more research to figure out. The objective of the study was to investigate how the taste was influenced by olfactory impairment in the central pathway. We tested 33 subjects with normal $(n=19)$ or impaired $(n=14)$ olfactory function for their gustatory event-related potentials (gERPs). Validated tests were used for olfactory and gustatory testing (Sniffin' Sticks, gERPs, and three-drop test). This study reported an objective gustatory function decline in olfactory dysfunction participants. However, it also reported the increased gustatory event-related potentials of olfactory dysfunction participants, especially at the frontal electrode $\left(\mathrm{F}_{\mathrm{Z}}\right)$ and electrode $16(\mathrm{E} 16)$, and the reduced latency of $\mathrm{P} 2$ peak of them at electrode 21 (E21), while no obvious difference was observed at the centro-parietal electrode $\left(\mathrm{P}_{\mathrm{Z}}\right)$. Inferior insula might be the main response area for the increase in gERPs, and this increase averaged amplitude of the P2 component may attribute to compensation of the secondary gustatory response that occurred in the gustatory processing of olfactory-impaired patients.
\end{abstract}

Keywords Gustatory event-related potentials $\cdot$ gERPs $\cdot$ Olfactory dysfunction $\cdot$ Odor $\cdot$ Taste

\section{Introduction}

Tastes and smells play important roles in life and provide a vital tool for screening soluble chemicals for food evaluation, selection, and avoidance of potentially toxic substances [1]. A high comorbidity rate of olfactory and

Zirong Chen and Chunhua Hu contributed equally to this work.

Yongxiang Wei

wei2203130113@163.com

1 Beijing Institute of Heart, Lung and Blood Vessel Diseases, Anzhen Road, Beijing, Chaoyang District, China

2 Department of Otolaryngology, Smell and Taste Center, Beijing Anzhen Hospital, Capital Medical University, Anzhen Road 2, Beijing, Chaoyang District 100010, China

3 Department of Psychiatry, National Clinical Research Center for Mental Disorders, China National Technology Institute On Mental Disorders, The Second Xiangya Hospital of Central South University, Changsha, Hunan, China

4 Department of Otorhinolaryngology-Head and Neck Surgery, Capital Institute of Pediatrics, Yabao Road 2, Chaoyang District, 100029 Beijing, China gustatory impairment was reported in the present studies $[2,3]$. This rate varies widely among studies, which was partly attributed to the difficulties of distinguishing gustatory from retro-nasal smell produced in the oral cavity and nasopharynx [4, 5]. Swiecicki L et al. provided information on the prevalence of smell and taste disorders in a non-clinical population, which estimates range from 2.7 to $76.8 \%$ and 5 to $20 \%$ [6]. And an estimated $95 \%$ of taste disorders which are caused by impairment of smell rather than the gustatory loss were reported [7]. Although these two systems identify and mediate different qualities and their fibers are not found intermingled with each other at any peripheral site, some daily works such as perceiving flavor require intricate integration of taste and olfactory information [8]. Taste and odor afferent information were confirmed to converge at the orbitofrontal cortex in the center [9].

Evidence showed a high comorbidity rate of olfactory and gustatory dysfunction is based on the overlap of taste and odor representation in the brain center. Traditional theories propose that the orbitofrontal cortex (OFC) is the first area capable of responding to both olfactory and gustatory stimuli $[10,11]$. The convergence of taste and smell information onto single neurons is realized in the caudal OFC (and 
immediately adjacent agranular insula) [10]. Recent studies consistently found that the gustatory cortex (GC) responds to both olfactory and gustatory stimuli. Taste perception have the ability to influence olfactory coding [12]. The insular cortex contributes to olfactory perception through overlapping taste and odor representation in it, which was based on the monosynaptic connections between the primary insular gustatory cortex and the primary piriform olfactory cortex [13]. These overlapping transduction mechanisms and connection pathways in the center proved that it is feasible for the olfactory function to affect gustatory function. However, those researches on the influences of smell on taste were mostly based on psychophysical chemical tests and functional imaging examinations. In psychophysical chemical tests, impaired olfactory function, especially the retro-nasal smell produced by volatile agents dissolved in the mouth by chewing and saliva, was usually roughly reported as changes in "taste perception" by subjects can lead to data distortion $[14,15]$. The utility of functional imaging examinations was restricted by the delayed cortical response to the taste stimuli and unclear brain positioning.

Gustatory event-related potentials (gERPs) had been proven a relatively unbiased, reliable, and easy approach to objectively assess human taste function [16]. It derives from electroencephalographic (EEG) measures and recorded the early cortical response to gustatory stimulus in milliseconds. In the previous study in our group, we have used it for exploring the characteristics of EEG to gustatory stimulus in the old [17]. In this study, we compared the gERPs between participants with olfactory dysfunction and volunteers with normal olfaction. And through the correlation analysis of olfaction and gERPs, we explore the potential effect of olfactory function on early central cortical response to the gustatory stimulus.

\section{Method and materials}

\section{Participants}

Adult patients over 50 years old were recruited from the Smell and Taste Center, Beijing Anzhen Hospital, Capital Medical University. All the participants underwent complete physical examination, nasal endoscopy, sinus computed tomography (CT), head magnetic resonance imaging (MRI), and Sniffin' Sticks tests. Demographics including age, sex, body mass index (BMI), smoking, and drinking were collected. Inclusion criteria included participants with normal gustatory function assessed by the three-drop test. Exclusion criteria included (1) patients with gustatory dysfunction with known causes (e.g., oral or dental morbidity, diabetes, neurological disorders, trauma, exposure to toxins or medications, congenital disorders, and idiopathic gustatory dysfunction); (2) participants diagnosed with cardiovascular disease, psychiatric disorder, or certain specific medical histories; (3) participants with tumor, cancer, or other chronic diseases that might influence the olfaction and cognition; and (4) participants without self-help skills. Subjects were not allowed to eat or smoke $2 \mathrm{~h}$ before the test. Finally, 33 participants were enrolled from the Smell and Taste Center of Beijing Anzhen Hospital, and they were divided into two groups (patients, 14 patients with the main complaint of olfactory dysfunction, and controls, 19 healthy volunteers). This study was approved by the Ethics Committee at Beijing Anzhen Hospital (Beijing, China, No. 2019YFE0116000). All participants provided written informed consent before participating in the study. The flow diagram of the study design was given in Fig. 1.

\section{Psychophysical olfactory test}

Psychophysical testing of olfactory function was performed for each participant using Sniffin' Sticks tests (Burghart, Gmbh, Wedel, Germany) which were consisted of odor threshold (OT), odor discrimination (OD), odor identification (OI), and the overall composite scores (TDI, a composite score of threshold, discrimination, and identification) [18]. Standard administration was performed according to the manufacturer's instructions [18]. Felt-tip pens containing various odors were presented to the participants for testing. The pen's tip was placed approximately $2 \mathrm{~cm}$ in front of both nostrils for bilateral stimulation. The test comprised three parts: OT, OD, and OI test. The overall results were combined and reported as TDI scores which ranged from 1 to 48 , with higher scores indicating superior olfactory performance. Subjects with a TDI score greater than 30 are considered to be normosmia; otherwise, they are considered to be hyposmia. A TDI score less than or equal to 15 points was defined as anosmia [18].

\section{Psychophysical gustatory test}

Briefly, eight different concentrations of $\mathrm{NaCl}$ (salty), sucrose (sweet), citric acid (sour), and quinine hydrochloride (bitter) solutions were prepared for this test, with the minimum concentrations of sucrose $(0.19 \mathrm{~g} / \mathrm{ml}), \mathrm{NaCl}$ $(0.06 \mathrm{~g} / \mathrm{ml})$, citric acid $(0.15 \mathrm{~g} / \mathrm{ml})$, and quinine hydrochloride $(0.0012 \mathrm{~g} / \mathrm{ml})[17,19-21]$. Tastants drops were dripped onto the middle of the anterior two-thirds of the extended tongue by pipettes [19]. Subjects were asked to describe the taste ("salty," "sweet," "sour," and "bitter"; multiple forced-choice tasks) and then rinse their mouths with tap water before the next tasks [17]. The score for each taste was ranged from 8 to 1 , and 1 represented the maximum concentration, while 8 represented the minimum concentration. The 


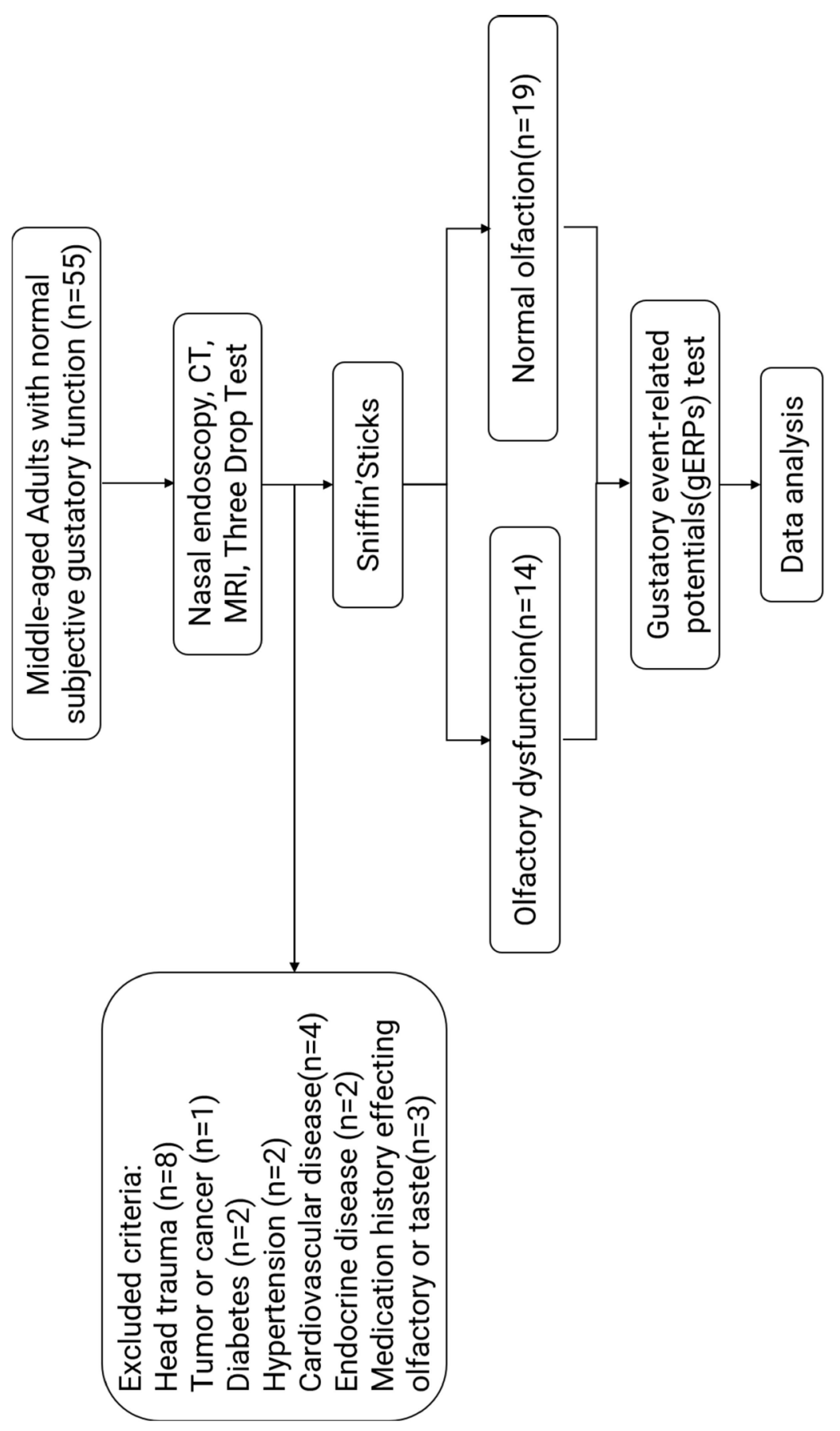


score was only recorded when the taste was identified twice consecutively [17].

\section{Electrophysiological gustatory test}

\section{Stimulation}

$\mathrm{NaCl}$ (tastant, $0.3 \mathrm{M}$ ) solution was used as the gustatory stimulus and distilled water for rinsing off tastants [22]. Gustatory stimulation method has previously been described in detail in our previous study, which featured a computercontrolled gustometer (Emerging Tech Trans LLC, Hershey, Pennsylvania, USA) that delivered solutions through peristaltic pumps and Teflon tubes [17]. Through two separate channels, at a rate of $39 \mathrm{ml} / \mathrm{min}$, liquids were kept at a similar temperature as the tongue $\left(36^{\circ} \mathrm{C}\right)$ and delivered into the mouth. And subjects were instructed to swallow or keep still by visual cues. A single cycle included five sessions, $2 \mathrm{~s}$ for relaxing, followed by $2 \mathrm{~s}$ for tasting, $5 \mathrm{~s}$ for stilling, $6 \mathrm{~s}$ for rinsing, and $5 \mathrm{~s}$ for swallowing in turn, and 60 cycles composed a test. Transistor-transistor logic pulses $(+5 \mathrm{~V}, \geq 10 \mathrm{~ms})$ were used to trace the stimulus of tastant or distilled water, which were marked by EEG DINS of " 1 " and " 2 " on the EEG recording software for different stimuli [17].

\section{Gustatory event-related potential recording and data analysis}

Continuous EEG was recorded following the international 10-20 system using a sensor net with scalp electrodes and amplified by the Net Amps 400 (Electrical Geodesics Inc., Eugene, Oregon, USA) [17]. Four sites were recorded by surface electrodes for statistical analysis, as described by the previous study [23]: centro-parietal electrode $\mathrm{P}_{Z}$, electrode 16 (E16), electrode 21 (E21), and frontal electrode $F_{Z}$. The detailed location of these electrodes was shown in Fig. 2. EEG data were processed by the MATLAB R2021a. GERPs from these four electrodes were analyzed and were proved representative of the cerebral gustative signal [16]. GERPs ( $n \geq 50)$ were averaged by software, and then the components were detected. Three components were defined by peaks with P1, N1, and P2 being the first (positive), second (negative), and third (positive) peaks, respectively. The latency was defined as the time interval between the stimulus and the peak. The amplitude was defined as the distance between the peak and the baseline [17]. Latencies (ms) and amplitudes $(\mu \mathrm{V})$ of each component were collected and analyzed. The detail of gERPs was shown in Fig. 3.

\section{Sample size calculation}

This study was a case-control study. The olfactory dysfunction group was the experimental group; the normal olfactory group was the control group. The amplitudes and latency of peaks (P1, N1, P2) were the main observation indicator in the present study. According to a similar case-control study about olfactory function in two groups [24], it was estimated that the average amplitude of P1 of the control group was $4.5 \pm 0.6 \mathrm{uV}$ and that of the experimental group was $3.6 \pm 0.4 \mathrm{uV}$. The difference between the two groups was $0.9 \mathrm{uV}$. Set $\alpha=0.05$ (two-sided), $\beta=0.10$, and $r=0.42$, and the sample size of the experimental group and the control group calculated by PASS 15.0.5 software was 8 (control group) and 11 (experimental group), respectively.

\section{Statistics analysis}

Statistical analysis was performed with Statistical Product and Service Solutions 22.0 (SPSS 22.0, IBM Corporation, New York, NY, USA). Shapiro-Wilk test was used to verify the normal distribution. Continuous variables were presented as mean \pm standard deviation (SD), and categorical variables were expressed as numerals (percentages). Two-tailed values of $p<0.05$ were considered statistically significant. Demographic data and psychophysical gustatory or olfactory tests from patients or controls are compared by the Chi-square test. Psychophysical gustatory or olfactory test scores between patients and controls were compared by independent $t$ test. Each component of gERPs (latency and amplitude from three peaks in three electrodes) between patients and controls was compared using an independent $t$ test. Spearman analysis was used to explore the correlation between gERPs and data of psychophysical of in 33 subjects $(r>0$ represents positive correlation, $r<0$ represents negative correlation, the closer $r$ value to 1 , the stronger correlation is).

\section{Results}

\section{Demographic data and psychophysical sensory tests}

Demographic characteristics and data of psychophysical sensory function tests of the above groups were recorded in Table 1. There were no significant differences in demographic data between patients and controls. None had dental or neurological problems or any remarkable medical history. Patients with olfactory impairment had a significantly lower odor threshold score, odor discrimination score, odor identification, and threshold discrimination-identification (TDI) score. As to the gustatory performance assessed by the three-drop test, there was only a significant difference of sweet scores between the patients $(5.40 \pm 2.12)$ and the controls $(7.37 \pm 1.38)$ which was observed. 
Fig. 2 Location of electrodes

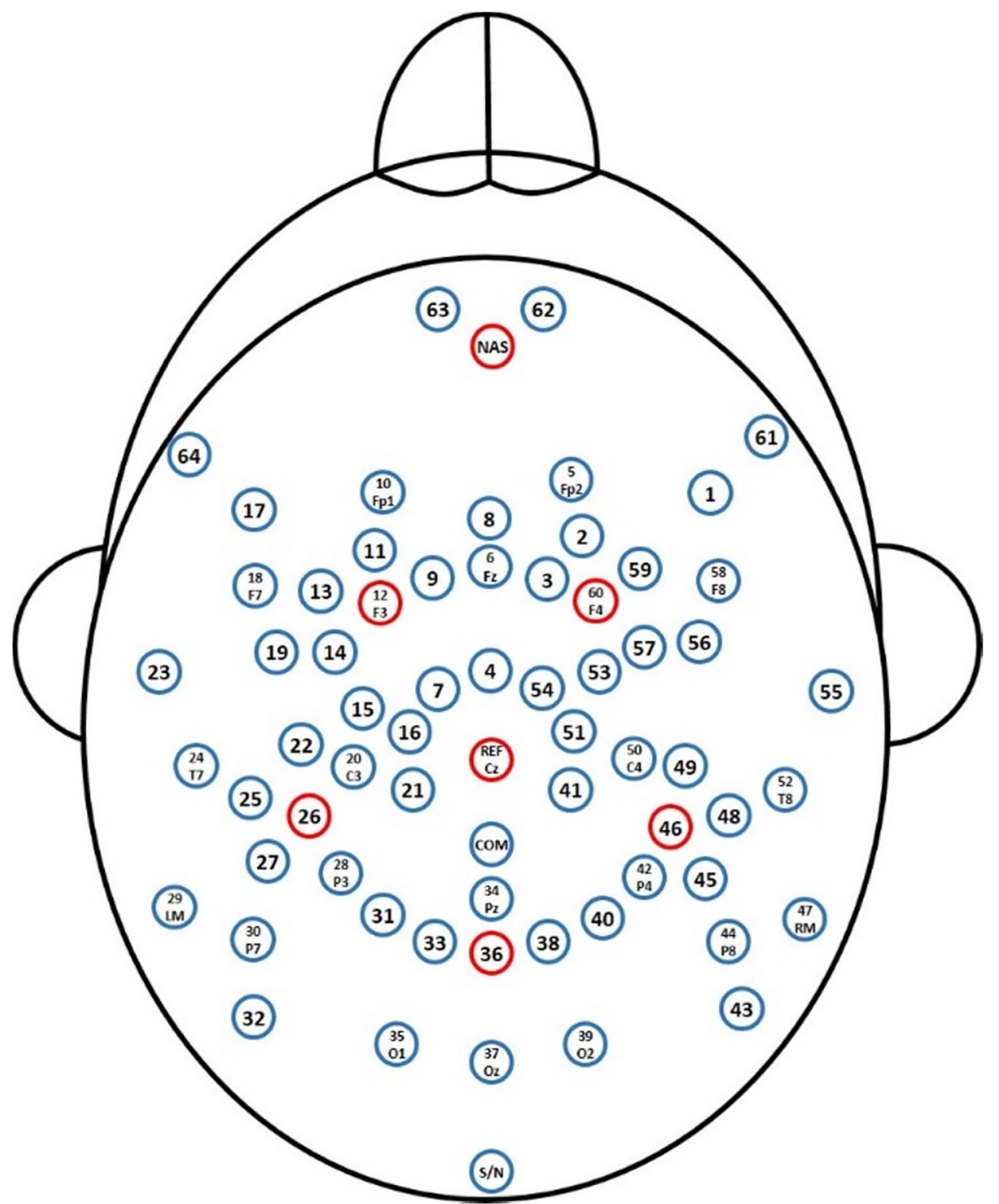

\section{Gustatory event-related potentials}

After averaging the gERPs recorded at four electrodes, three peaks (P1, N1, and P2) were observed for all participants. The mean value and standard deviation (SD) of latencies and amplitudes of P1, N1, and P2 of patients and controls were detailed in Table 2 and Fig. 3. To confirm that the gERPs were uniquely evoked by tastants, we examined a sequence of the potentials evoked by distilled water and found no significant peaks.

No significant difference was observed for P1 averaged latency and amplitude between groups. A significant difference can be observed at E16 $(p=0.033)$ for $\mathrm{N} 1$ averaged amplitude between groups. The patient group $(-2.55 \pm 1.92 \mu \mathrm{V})$ demonstrated a larger amplitude of the $\mathrm{N} 1$ component compared to the control group
$(-1.06 \pm 1.58 \mu \mathrm{V})$. A significant difference can be observed at $\mathrm{F}_{\mathrm{Z}}$ for the $\mathrm{P} 2$ averaged amplitude between groups $(p=0.000)$. The patient group $(5.63 \pm 1.59 \mu \mathrm{V})$ demonstrated a larger amplitude of $\mathrm{P} 2$ component compared to the control group $(2.32 \pm 2.42 \mu \mathrm{V})$. The difference of P2 averaged latency between groups can be observed at the E21 $(p=0.040)$, and the control group $(244.86 \pm 25.40 \mathrm{~ms})$ showed a longer latency compared to the patient group $(226.17 \pm 18.38 \mathrm{~ms})$. All the details were recorded in Table 2 .

\section{Correlation analysis}

The subjective gustatory performance of sweet showed a significant correlation with olfactory performance, which was listed in Table 3. The details of correlation analysis for 

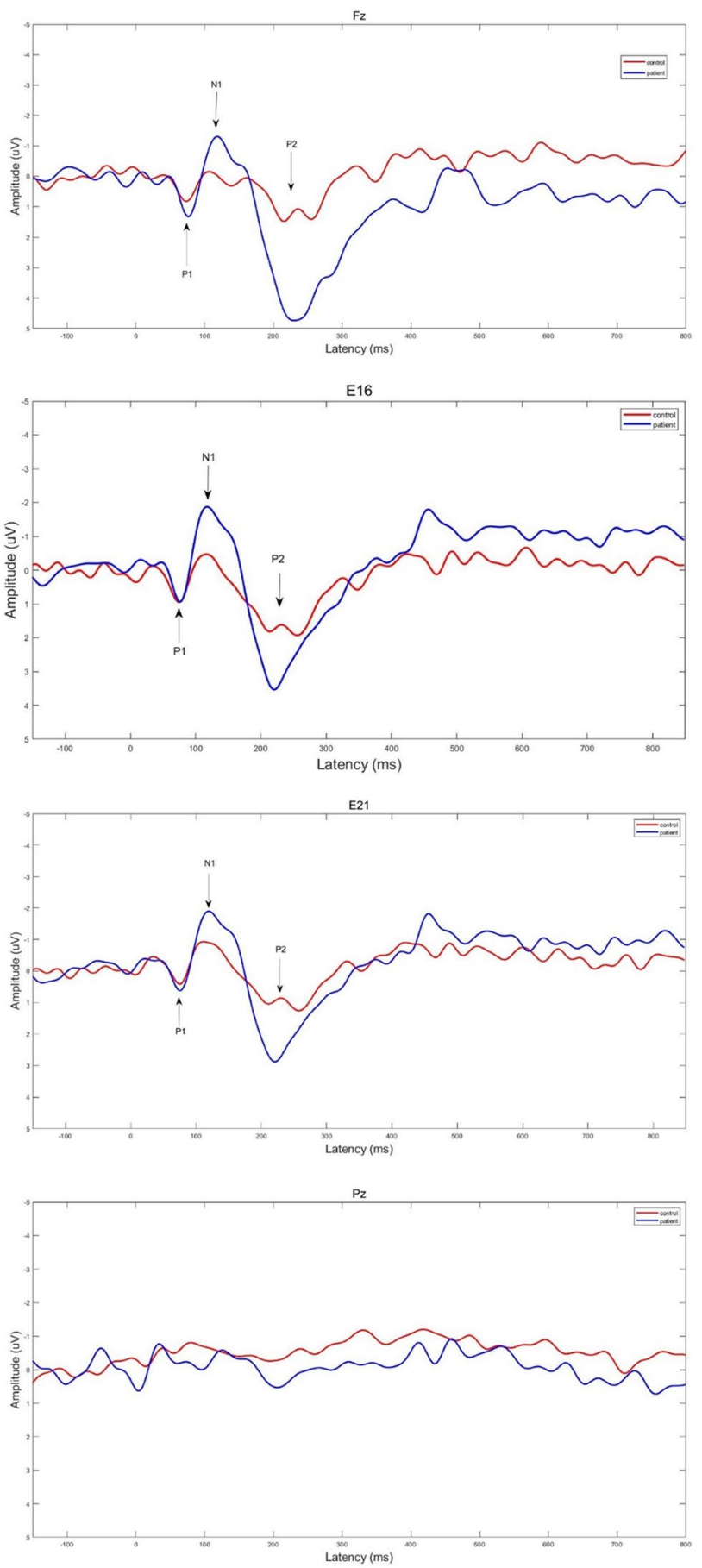

Fig. 3 Grand averaged latencies and amplitudes of gustatory eventrelated potentials for patients and controls

gERPs and psychophysical olfactory test scores (TDI) were demonstrated in Table 4.

After the adjustment for age and gender, we observed a significant correlation between P1 averaged amplitude and odor threshold scores ( $\mathrm{T}, r=0.423, p=0.035)$ at E16. The P1 averaged amplitude increased with the higher olfactory
Table 1 Characteristics of participants

\begin{tabular}{llll}
\hline & Patients & Controls & $P$ \\
\hline$N$ (female\%) & $14(64.29)$ & $19(84.21)$ & $\mathrm{ns}$ \\
Age (year) & $50.92 \pm 9.69$ & $48.37 \pm 4.84$ & 0.331 \\
BMI $\left(\mathrm{kg} / \mathrm{m}^{2}\right)$ & $23.57 \pm 1.31$ & $24.74 \pm 2.13$ & 0.166 \\
Smoking & 0 & 0 & - \\
Drinking & 0 & 0 & - \\
Hypertension & 0 & 0 & - \\
Diabetes & 0 & 0 & - \\
PTOD & 5 & 0 & - \\
PVOD & 8 & 0 & - \\
Idiopathic OD & 1 & 0 & - \\
Salty & $6.40 \pm 2.11$ & $6.16 \pm 1.54$ & 0.726 \\
Sweet & $5.40 \pm 2.12$ & $7.37 \pm 1.38$ & $0.003^{* * *}$ \\
Sour & $6.70 \pm 1.57$ & $6.95 \pm 1.22$ & 0.642 \\
Bitter & $7.00 \pm 1.63$ & $8.00 \pm 1.20$ & 0.071 \\
T & $1.61 \pm 1.21$ & $13.77 \pm 2.51$ & $0.000^{* * *}$ \\
D & $4.93 \pm 2.79$ & $10.32 \pm 1.77$ & $0.000^{* * *}$ \\
I & $6.29 \pm 3.89$ & $12.42 \pm 1.57$ & $0.000^{* * *}$ \\
TDI & $12.68 \pm 7.33$ & $36.39 \pm 3.65$ & $0.000^{* * *}$ \\
\hline
\end{tabular}

$P T O D$, post-viral olfactory dysfunction; $P V O D$, post-traumatic olfactory dysfunction; $O D$, olfactory dysfunction. *, difference is significant at 0.05 level; $n s$, non-statistically significant

performance. For the N1 peak, we also observed a significant correlation between the averaged amplitude and odor threshold scores (T, $r=0.476, p=0.016$ ) at E16. For the P2 peak, at frontal electrode $\mathrm{F}_{\mathrm{Z}}$, we observed a significant correlation between the averaged amplitude and olfactory test scores (T, $r=-0.417, p=0.038 ; \mathrm{D}, r=-0.497, p=0.011$; I, $r=-0.463, p=0.020$; and TDI, $r=-0.478, p=0.016$ ). At E16, the averaged latency of $\mathrm{P} 2$ peak showed significant correlations with olfactory test scores $(\mathrm{T}, r=0.421, p=0.036$; $\mathrm{I}, r=0.564, p=0.003$; TDI, $r=0.474, p=0.017$ ). At E21, the $\mathrm{P} 2$ averaged latency also reported significant correlations with olfactory test scores (T, $r=0.444, p=0.026$; D, $r=0.436, p=0.029$; I, $r=0.602, p=0.001$; TDI, $r=0.523$, $p=0.007)$. The $\mathrm{P} 2$ averaged amplitude increased with the impaired olfactory performance, while the averaged latency increased with the rising olfactory performance.

\section{Discussion}

The most obvious difference of gERPs' components between the patients and the controls was observed at E16, E21, and the frontal electrode $\mathrm{F}_{\mathrm{Z}}$. The averaged amplitude of the P1 component of gERPs reported a positive correlation to olfactory performance, while for $\mathrm{P} 2$ component, it demonstrated an opposite tendency to olfactory performance. And even 
Table 2 Grand averaged latencies and amplitudes of gustatory event-related potentials for patients and controls

\begin{tabular}{|c|c|c|c|c|c|c|c|}
\hline \multirow{2}{*}{\multicolumn{2}{|c|}{$\begin{array}{l}\text { Gustatory } \\
\text { ERPs }\end{array}$}} & \multicolumn{3}{|l|}{ Latency $(\mathrm{ms})$} & \multicolumn{3}{|l|}{ Amplitude $(\mu \mathrm{v})$} \\
\hline & & Patients $(n=14)$ & Controls $(n=19)$ & $\mathrm{P}$ & Patients $(n=14)$ & Controls $(n=19)$ & $P$ \\
\hline \multirow[t]{3}{*}{$\mathrm{F}_{\mathrm{Z}}$} & $\mathrm{P} 1$ & $87.17 \pm 26.92$ & $96.88 \pm 33.91$ & ns & $1.85 \pm 1.41$ & $1.87 \pm 1.65$ & ns \\
\hline & N1 & $131.00 \pm 18.98$ & $142.63 \pm 32.11$ & ns & $-2.12 \pm 2.21$ & $-1.37 \pm 1.74$ & ns \\
\hline & $\mathrm{P} 2$ & $244.5 \pm 27.98$ & $227.00 \pm 22.74$ & $\mathrm{~ns}$ & $5.63 \pm 1.59$ & $2.32 \pm 2.42$ & $0.000^{* *}$ \\
\hline \multirow[t]{3}{*}{ E16 } & $\mathrm{P} 1$ & $85.67 \pm 24.85$ & $100.00 \pm 38.82$ & $\mathrm{~ns}$ & $1.12 \pm 0.94$ & $1.75 \pm 1.73$ & ns \\
\hline & $\mathrm{N} 1$ & $123.17 \pm 16.94$ & $136.5 \pm 31.09$ & $\mathrm{~ns}$ & $-2.55 \pm 1.92$ & $-1.06 \pm 1.58$ & $0.033^{*}$ \\
\hline & $\mathrm{P} 2$ & $226.33 \pm 17.54$ & $241.25 \pm 24.59$ & ns & $4.07 \pm 1.77$ & $2.78 \pm 3.00$ & ns \\
\hline \multirow[t]{3}{*}{ E21 } & $\mathrm{P} 1$ & $80.50 \pm 22.40$ & $91.50 \pm 34.86$ & ns & $0.95 \pm 0.83$ & $1.30 \pm 1.66$ & ns \\
\hline & N1 & $130.33 \pm 20.00$ & $131.62 \pm 26.90$ & ns & $-2.62 \pm 1.87$ & $-1.75 \pm 1.40$ & ns \\
\hline & $\mathrm{P} 2$ & $226.17 \pm 18.38$ & $244.86 \pm 25.40$ & $0.040^{*}$ & $3.44 \pm 1.54$ & $2.26 \pm 3.10$ & ns \\
\hline \multirow[t]{3}{*}{$\mathrm{P}_{\mathrm{Z}}$} & $\mathrm{P} 1$ & $99.33 \pm 33.19$ & $109.38 \pm 34.52$ & ns & $0.75 \pm 0.77$ & $0.55 \pm 1.28$ & ns \\
\hline & N1 & $131.17 \pm 20.86$ & $139.88 \pm 37.94$ & ns & $8.12 \pm 32.40$ & $-1.47 \pm 1.06$ & ns \\
\hline & $\mathrm{P} 2$ & $230.83 \pm 32.36$ & $240.75 \pm 34.50$ & $\mathrm{~ns}$ & $1.17 \pm 1.26$ & $0.51 \pm 1.54$ & $\mathrm{~ns}$ \\
\hline
\end{tabular}

*the difference is significant at 0.05 level; **the difference is significant at 0.01 level. $F_{Z}$, the frontal electrode; $C_{Z}$, the central electrode; $\mathrm{P}_{\mathrm{Z}}$, the centro-parietal electrode
Table 3 Correlation analysis for sweet score and olfactory performance (TDI)

\begin{tabular}{llllll}
\hline Correlation & & $\mathrm{T}$ & $\mathrm{D}$ & $\mathrm{I}$ & $\mathrm{TDI}$ \\
\hline Sweet & $\mathrm{r}$ & $0.501^{* *}$ & $0.497^{* *}$ & $0.407^{*}$ & $0.499^{* *}$ \\
& $\mathrm{p}$ & 0.006 & 0.006 & 0.028 & 0.006 \\
\hline
\end{tabular}

** correlation is significant at the 0.01 level (2-tailed)

* correlation is significant at the 0.05 level (2-tailed)

the averaged latency of the $\mathrm{P} 2$ peak became longer with the increased odor scores.

A significant correlation between TDI scores and sweet scores was observed in this study, which was in line with gustatory complaints of patients with olfactory impairment in daily life. In previous studies, it was widely accepted that olfactory impairment patients would report a light gustatory decline, especially in the old, the patients with neurodegenerative diseases such as PD or AD, the patients with psychological diseases such as autism, and upper airway infection diseases such as COVID-19 [25-30]. Patients with olfactory dysfunction after upper respiratory tract infection reported that the degree of damage to the taste function was increased with the more severe the damage of olfactory function [31]. Behavioral studies confirmed that congruent odors could amplify taste intensity [32-34]. In daily life, people tend to confuse the "flavor" with the taste or the gustatory, but the flavor is the combination of taste and smell sense especially the retro-nasal olfactory sense when smells arise from inside the mouth during consumption, stimulating the epithelium upon exhalation [10,35]. We believe that retro-nasal olfactory function should be responsible for some part of the complaint about gustatory function. Retro-nasal odors were reported to share processing circuitry commonly associated with taste, and the input was processed by a brain region responsible for taste processing, which was known as the insular gustatory cortex [35]. From previous studies, the insular cortex contributed to odor quality coding by representing the taste-like aspects of food odors. And odorized air activated the piriform cortex, OFC, and insula, among which, only the insular cortex responded to sweet taste [36]. The decreased sweet scores for olfactory-impaired patients
Table 4 Correlation analysis for gERPs (latency and amplitude) and olfactory performance

\begin{tabular}{|c|c|c|c|c|c|c|c|c|}
\hline \multirow[t]{2}{*}{ Correlation } & \multicolumn{2}{|l|}{$\mathrm{T}$} & \multicolumn{2}{|l|}{$\mathrm{D}$} & \multicolumn{2}{|l|}{ I } & \multicolumn{2}{|l|}{ TDI } \\
\hline & $r$ & $p$ & $r$ & $p$ & $r$ & $p$ & $r$ & $p$ \\
\hline $\mathrm{F}_{\mathrm{Z}} \mathrm{P} 2-\mathrm{A}$ & $-0.417^{*}$ & 0.038 & $-0.497^{*}$ & 0.011 & $-0.463^{*}$ & 0.020 & $-0.478^{*}$ & 0.016 \\
\hline E16P1-A & $0.423^{*}$ & 0.035 & 0.084 & 0.691 & 0.134 & 0.524 & 0.265 & 0.201 \\
\hline E16N1-A & $0.476^{*}$ & 0.016 & 0.023 & 0.907 & 0.161 & 0.414 & 0.264 & 0.175 \\
\hline E16P2-L & $0.421^{*}$ & 0.036 & 0.351 & 0.086 & $0.564^{* *}$ & 0.003 & $0.474^{*}$ & 0.017 \\
\hline E21P2-L & $0.444^{*}$ & 0.026 & $0.436^{*}$ & 0.029 & $0.602^{* *}$ & 0.001 & $0.523^{\text {** }}$ & 0.007 \\
\hline
\end{tabular}

*the difference is significant at 0.05 level; **the difference is significant at 0.01 level. $F_{Z}$, the frontal electrode; $C_{Z}$, The central electrode; $P_{Z}$, the centro-parietal electrode. $O T$, odor threshold; $O D$, odor discrimination; $O I$, odor identification; $T D I$, composite score of threshold, discrimination, and identification 
may be attributed to the damage in overlapping regions of the insular cortex. A study explored the eating behavior of olfactory-impaired patients which also reported patients tended to use more sugar [37]. Impaired olfactory function weakened gustatory function gradually through the insular cortex, which was reflected on the sweet score.

A significant correlation between TDI scores and gERPs (including amplitudes and latencies) can be reported in this study. For the P1 and N1 components, their averaged amplitudes increased with odor threshold scores. Previous studies hold that the P1 component was the first gustatory response, namely, at around $100 \mathrm{~ms}$, which was located in the transition between temporal operculum and insula, while the N1 peak was located in the middle insula, and they seem to reflect the activity of the primary gustatory area [38, 39]. And the fronto-central maximum for early amplitudes P1 (and also N1) was considered to relate specifically to gustatory function [16]. A Faurion et al. held that N1 was determined by exogenous stimulus properties (concentration or quality) [39]. In this study, averaged amplitudes of P1 and N1 decreased with the impaired olfaction, which may reflect a slight adverse effect on the primary gustatory area related to impaired olfaction. But the $\mathrm{P} 2$ component turned out a different tendency compared to the P1 component and the sweet score. For the P2 averaged amplitude recorded at $\mathrm{F}_{\mathrm{Z}}$, the patient group reported a huger one than the control group. For the averaged latency, P2 was in a line with the increased olfactory performance, especially at E16. Olfactory-impaired patients reported a relatively huge averaged amplitude of $\mathrm{P} 2$, which is seemly opposed to normal speculates and the complaint of patients. Chie Mizoguchi et al. proposed P2 was commonly associated with the inferior insula, whose region was likely to be involved in gustatory cognitive processing, and it reflected higher gustatory functions [22]. In early studies, the posterior part of the insula has a role of more secondary gustatory response related to the subjective interception of the stimulus, and the P2 component may be dependent on endogenous processes reflecting the subjective significance of the stimuli, which could be in line with the late positive component (LPC) in olfactory event-related potential [39]. A study exploring the olfactory event-related potential of mild cognitive impairment patients found N1 amplitude was reduced, while the LPC amplitude was increased, and it proposed that a degree of perceptive compensation can occur when a sensorial function was impaired [40]. We reasonably speculated that a compensation mechanism works in the gustatory sensory system; a degree of compensation of the secondary gustatory response can occur in gustatory processing of olfactoryimpaired patients, which was reflected on the $\mathrm{P} 2$ component.

Furthermore, A Faurion et al. reported a significant lateralized integration between gustatory and olfactory information, in the inferior part of the insula, which may be interpreted as a second-order gustatory area related to secondary processing [41]. Another PET study also reported a left insular activation in response to gustatory stimulation for right-handers [42]. In this study, we also noticed the phenomenon that the gEPRs recorded at the left showed the more obvious components of gEPRs compared to the records at the right, which supported the previous concept of lateralized integration between gustatory and olfactory information.

\section{Conclusion}

In this study, we explored not only the difference of three components of gERPs between the olfactory dysfunction population and normal olfactory function adults but also the association between gustatory and olfactory function. The P1 amplitudes reflected primary gustatory area which was decreased with the declined olfactory performance, while the P2 amplitudes related to the secondary gustatory processing or higher gustatory processing increased with better olfactory performance. These findings are conducive to explore the impairment of gustatory dysfunction in the olfactory dysfunction population, which may give more information about the interaction of gustatory and olfactory function.

Author contribution All authors have made substantial contributions to the conception, analysis, and interpretation of data in this article, approved the submitted version, and agreed both to be personally accountable for our contributions and to ensure that questions related to the accuracy or integrity of any part of the work, even ones in which we are not personally involved, are appropriately investigated, resolved, and the resolution documented in the literature.

Funding This study is supported by grants from the Key Special Program for International Science and Technology Cooperation Program of China (SQ2019YFE011488).

\section{Declarations}

Ethical approval This study was approved by the Ethics Committee at Beijing Anzhen Hospital (Beijing, China, No. 2019YFE0116000). The study design complied with the criteria of the Declaration of Helsinki for Medical Research involving Human Subjects. All participants provided written informed consent before participating in the study.

Conflict of interest The authors declare no competing interests.

\section{References}

1. Maheswaran T, Abikshyeet P, Sitra G, Gokulanathan S, Vaithiyanadane V, Jeelani S (2014) Gustatory dysfunction. (0976-4879). https://doi.org/10.4103/0975-7406.137257. 
2. Landis BN, Scheibe M, Weber C, Berger R, Brämerson A, Bende M, Nordin S, Hummel T (2010) Chemosensory interaction: acquired olfactory impairment is associated with decreased taste function. J Neurol 257(8):1303-1308. https://doi.org/10.1007/ s00415-010-5513-8

3. Gudziol H, Rahneberg K, Burkert S (2007) Anosmics are more poorly able to taste than normal persons. Laryngo-Rhino-Otologie 86(9):640-643. https://doi.org/10.1055/s-2007-966228

4. Suen JLK, Yeung AWK, Wu EX, Leung WK, Tanabe HC, Goto TK (2021) Effective connectivity in the human brain for sour taste, retronasal smell, and combined flavour. Foods (Basel, Switzerland) 10(9). 10.3390/foods10092034.

5. Negoias S, Meves B, Zang Y, Haehner A, Hummel T (2020) Characteristics of olfactory disorder with and without reported flavor loss. Laryngoscope 130(12):2869-2873. https://doi.org/10.1002/ lary. 29070

6. Doty RL (2019) Epidemiology of smell and taste dysfunction. Handbook of clinical neurology. 164. https://doi.org/10.1016/ B978-0-444-63855-7.00001-0.

7. Malaty J, Malaty IA (2013) Smell and taste disorders in primary care. Am Fam Physician 88(12):852-859

8. Bromley SM, Doty RL (2019) Health histories and medical evaluations of patients with complaints of chemosensory dysfunction. Handb Clin Neurol 164:219-227. https://doi.org/10.1016/B9780-444-63855-7.00014-9

9. Rolls ET (2005) Taste, olfactory, and food texture processing in the brain, and the control of food intake. Physiol Behav 85(1):45-56

10. de Araujo IET, Rolls ET, Kringelbach ML, Mcglone F, Phillips $\mathrm{N}$ (2003) Taste-olfactory convergence, and the representation of the pleasantness of flavour, in the human brain. Eur J Neurosci 18(7):2059-2068. https://doi.org/10.1046/j.1460-9568.2003. 02915.x

11. Rolls ET, Baylis LL (1994) Gustatory, olfactory, and visual convergence within the primate orbitofrontal cortex. J Neurosci Off J Soc Neurosci 14(9):5437-5452. https://doi.org/10.1523/JNEUR OSCI.14-09-05437.1994

12. Wilson DA, Stevenson RJ (2003) Olfactory perceptual learning: the critical role of memory in odor discrimination. Neurosci Biobehav Rev 27(4):307-328. https://doi.org/10.1016/s01497634(03)00050-2

13. Mesulam M, Mufson E (1985) The insula of Reil in man and monkey. Cereb Cortex 4:179-225. https://doi.org/10.1007/ 978-1-4757-9619-3_5

14. Pieniak M, Oleszkiewicz A, Klockow M, Yoshino A, Haehner A, Hummel T (2021) q-Powders: a quick test for screening retronasal olfactory disorders with tasteless powders. Euro Arch OtoRhino-Laryngol. https://doi.org/10.1007/s00405-021-06849-8.

15. Nettore IC, Maione L, Desiderio S, De Nisco E, Franchini F, Palatucci G, Ungaro P, Cantone E, Macchia PE, Colao A (2020) Influences of age, sex and smoking habit on flavor recognition in healthy population. International journal of environmental research and public health. 17(3). https://doi.org/10.3390/ijerp h17030959.

16. Hummel T, Genow A, Landis BN (2009) Clinical assessment of human gustatory function using event related potentials. J Neurol Neurosurg Psychiatry 81((4)(1468-330X)):459-464. https://doi. org/10.1136/jnnp.2009.183699

17. Hu C, Yao L, Chen Z, Sun Z, Zhang L, Wei Y (2020) Assessment of gustatory function in aging people using event-related potentials. NeuroReport 31(9):650-656. https://doi.org/10.1097/WNR. 0000000000001457

18. Hummel T, Sekinger B, Wolf SR, Pauli E, Kobal G (1997) "Sniffin" sticks': olfactory performance assessed by the combined testing of odor identification, odor discrimination and olfactory threshold. Chem Senses 22(1):39-52. https://doi.org/10.1093/ chemse/22.1.3
19. Henkin RI, Gill JR, Bartter FC (1963) Studies on taste thresholds in normal man and in patients with adrenal cortical insufficiency: the role of adrenal cortical steroids and of serum sodium concentration. J Clin Invest 42(5):727-735. https://doi.org/10. 1172/JCI104765

20. Henkin RI (1974) Salt taste in patients with essential hypertension and with hypertension due to primary hyperaldosteronism. J Chronic Dis 27(4):235-244. https://doi.org/10.1016/ 0021-9681(74)90048-4

21. Gudziol H, Hummel T (2007) Normative values for the assessment of gustatory function using liquid tastants. Acta Otolaryngol 127(6):658-661. https://doi.org/10.1080/000164806009514 91

22. Mizoguchi C, Kobayakawa T, Saito S, Ogawa H (2002) Gustatory evoked cortical activity in humans studied by simultaneous EEG and MEG recording. Chem Senses 27(7):629-634. https://doi.org/ 10.1093/chemse/27.7.629

23. Jacquin-Piques A, Gaudillat S, Mouillot T, Gigot V, Meillon S, Leloup C, Penicaud L, Brondel L (2015) Prandial states modify the reactivity of the gustatory cortex using gustatory evoked potentials in humans. Front Neurosci 9:490. https://doi.org/10. 3389/fnins.2015.00490

24. Hummel T, Haenel T, Pauli E, West W, Zahnert T, Hull D (2007) Investigations in subjects with cough following upper respiratory tract infection. Respir Physiol Neurobiol 156(1):79-84

25. Spinato G, Fabbris C, Polesel J, Cazzador D, Borsetto D, Hopkins C, Boscolo-Rizzo P (2020) Alterations in smell or taste in mildly symptomatic outpatients with SARS-CoV-2 infection. JAMA 323(20):2089-2090. https://doi.org/10.1001/jama.2020.6771

26. Bennetto L, Kuschner ES, Hyman SL (2007) Olfaction and taste processing in autism. Biol Psychiat 62(9):1015-1021. https://doi. org/10.1016/j.biopsych.2007.04.019

27. Tarakad A, Jankovic J (2017) Anosmia and ageusia in Parkinson's disease. Int Rev Neurobiol 133:541-556. https://doi.org/10.1016/ bs.irn.2017.05.028

28. Lang CJ, Leuschner T, Ulrich K, Stossel C, Heckmann JG, Hummel T (2006) Taste in dementing diseases and parkinsonism. J Neurol Sci 248(1-2):177-184. https://doi.org/10.1016/j.jns.2006. 05.020

29. Churnin I, Qazi J, Fermin CR, Wilson JH, Payne SC, Mattos JL (2019) Association between olfactory and gustatory dysfunction and cognition in older adults. Am J Rhinol Allergy 33(2):170 177. https://doi.org/10.1177/1945892418824451

30. Doty RL, Hawkes CH (2019) Chemosensory dysfunction in neurodegenerative diseases. Handb Clin Neurol 164:325-360. https:// doi.org/10.1016/B978-0-444-63855-7.00020-4

31. Li KY, Liu J, Xiao W, Wu Y, Ren YY, Wei YX (2016) Characteristics of postviral olfactory disorder. Zhonghua er bi yan hou tou jing wai ke za zhi 51(11):838-841. https://doi.org/10.3760/cma.j. issn.1673-0860.2016.11.007

32. Alison F. Bingham, Gordon G. Birch, de Graaf C, John M. Behan, K.D. Perring (1990) Sensory studies with sucrose-maltol mixtures. Chemical Senses 15(4), 447-456. https://doi.org/10.1093/ chemse/15.4.447

33. Djordjevic J, Zatorre RJ, Jones-Gotman M (2004) Odor-induced changes in taste perception. Exp Brain Res 159(3):405-408. https://doi.org/10.1007/s00221-004-2103-y

34. Schifferstein HN, Verlegh PW (1996) The role of congruency and pleasantness in odor-induced taste enhancement. Acta psychologica, 94(1). https://doi.org/10.1016/0001-6918(95)00040-2.

35. Blankenship ML, Grigorova M, Katz DB, Maier JX (2019) Retronasal odor perception requires taste cortex, but orthonasal does not. Current biology : CB, 29(1). https://doi.org/10.1016/j.cub. 2018.11.011.

36. Veldhuizen MG, Nachtigal D, Teulings L, Gitelman DR, Small DM (2010) The insular taste cortex contributes to odor 
quality coding. Frontiers in human neuroscience, 4. https://doi. org/10.3389/fnhum.2010.00058

37. Zang Y, Han P, Burghardt S, Knaapila A, Schriever V, Hummel $T$ (2019) Influence of olfactory dysfunction on the perception of food. Eur Arch Oto-Rhino-L 276(10):2811-2817. https://doi.org/ 10.1007/s00405-019-05558-7

38. Gotow N, Kobayakawa T (2019) Trial measurement of brain activity underlying olfactory-gustatory synchrony perception using event-related potentials from five female participants. J Neurosci Res 97(3):253-266. https://doi.org/10.1002/jnr.24310

39. Faurion A, Cerf B, Le Bihan D, Pillias AM (1998) fMRI study of taste cortical areas in humans. Ann N Y Acad Sci 855:535-545

40. Invitto S, Piraino G, Ciccarese V, Carmillo L, Caggiula M, Trianni G, Nicolardiu G, Di Nuovo S, Balconi M (2018) Potential role of OERP as early marker of mild cognitive impairment. Front Aging Neurosci 10. https://doi.org/10.3389/fnagi.2018.00272
41. Faurion A, Cerf B, Van De Moortele PF, Lobel E, Mac Leod P, Le Bihan D (1999) Human taste cortical areas studied with functional magnetic resonance imaging: evidence of functional lateralization related to handedness. Neuroscience Letters, 277(3), 189-192

42. Kinomura S, Kawashima R, Yamada K, Ono S, Itoh M, Yoshioka S, Yamaguchi T, Matsui H, Miyazawa H, Itoh H (1994) Functional anatomy of taste perception in the human brain studied with positron emission tomography. Brain Res 659(1-2):263-266

Publisher's note Springer Nature remains neutral with regard to jurisdictional claims in published maps and institutional affiliations. 